\title{
Vaginal polyp: an unusual cause of postmenopausal bleeding
}

\author{
Janaki Putran • Rachana Gupta
}

Received: 14 December 2009 / Accepted: 4 January 2010/Published online: 5 February 2010

(C) Springer-Verlag 2010

\begin{abstract}
Postmenopausal bleeding (PMB) can be due to various causes. The commonest cause is atrophy of the vagina or the endometrium. Endometrial cancer accounts for $10 \%$ cases of PMB. We present this unusual case of fibroepithelial polyp of the vagina as a cause for PMB. Vaginal fibroepithelial polyp is a rare lesion, and although benign, it can be confused with malignant connective tissue lesions. Treatment is simple excision, and recurrence is extremely uncommon.
\end{abstract}

Keywords Fibroepithelial polyp · Postmenopausal bleeding $\cdot$ Vagina

\section{Case report}

A 67-year-old woman presented with a history of postmenopausal bleeding (PMB) for 2 months. She suffered from type 1 diabetes and essential hypertension. She had a past history of breast carcinoma and was on tamoxifen. On examination, her body mass index (BMI) was 41. Abdominal examination was inconclusive and vaginal examination not possible as she was a virgo intacta. An abdominal ultrasound demonstrated a $7 \times 4-\mathrm{cm}$-sized retroverted uterus with endometrial thickness of $11 \mathrm{~mm}$. She was booked for examination under anesthesia and hysteroscopy. This was difficult due to poor access due to body habitus and a small introitus. A $1 \times 2-\mathrm{cm}$ friable growth was noted in the anterior vaginal wall $2 \mathrm{~cm}$ above the external urethral

J. Putran $(\bowtie) \cdot$ R. Gupta

Department of Obstetrics and Gynaecology,

Colchester Hospital University NHS Foundation Trust,

Turner Road,

Colchester CO4 5JL, UK

e-mail: jputran@hotmail.com orifice. The mass was removed and sent for urgent histology. Cervix was healthy. Saline hysteroscopy, endometrial biopsy, and subsequent histology were normal with no evidence of endometrial pathology.

The vaginal bleeding had settled when the woman was reviewed 6 weeks and 6 months after the surgery. The vaginal lesion was subsequently diagnosed as benign fibroepithelial polyp on histology.

\section{Discussion}

PMB represents one of the most common reasons for referral to gynecological services, largely due to suspicion of an underlying endometrial malignancy. Possible causes in order of frequency include atrophic endometritis and vaginitis, exogenous estrogens, endometrial carcinoma, endometrial or cervical polyps, endometrial hyperplasia, cervical carcinoma, ring pessary, and ovarian estrogensecreting tumors.

Endometrial cancer is present in approximately $10 \%$ of patients referred with PMB. In the above woman, the clinical suspicion for endometrial carcinoma was high. She was hence booked for urgent hysteroscopy and endometrial biopsy. However, the final cause of the PMB was the fibroepithelial polyp in the vagina.

Fibroepithelial polyp of the vagina was first described by Norris and Taylor in 1966. Although benign, it can be confused with malignant connective tissue lesions because of its bizarre histology. They typically occur in women of reproductive age but may be seen in postmenopausal women specially those on hormone replacement therapy [1].

The mean age at diagnosis is approximately 40 years, with an age range varying from that of the newborn child to 77 years. Fibroepithelial polyp as a cause of PMB is rare [2, 3]. 
Fibroepithelial polyps may develop as a result of a granulation tissue reaction after some local injury of the vaginal mucosa with hormonal factors modulating the growth $[4,5]$. Delayed differentiation of myofibroblastic cells may explain why granulation tissue sometimes does not contract properly but turn into polyps.

Fibroepithelial stromal polyps of the vault after hysterectomy are an uncommon, though well-recognized finding. Both tibolone and tamoxifen have been reported to cause polyps in the endometrium and their influence on the formation of vaginal polyps is increasingly being recognized.

Histologically, fibroepithelial polyps are composed of a squamous epithelial surface with a fibrovascular stalk and edematous stroma [6]. The lesions, when bland or hypocellular, are readily recognized as benign. However, those that exhibit bizarre cytomorphology, atypical mitoses, or hypercellularity may be confused with sarcoma botryoides, rhabdomyosarcoma, and mixed mesodermal tumor [7].

Clinically, the polyps are usually asymptomatic and are discovered incidentally during pelvic examination. They commonly occur on the lateral wall of the lower third of the vagina. The lesions are usually small and solitary, polypoid or pedunculated, and measuring 0.5 to $4 \mathrm{~cm}$ in size. Multiple lesions are known in pregnancy. During pregnancy, these lesions may become enlarged, very edematous, and bizarre in appearance.

Symptoms and signs may include a sensation of pressure, dyspareunia, obstruction of the vagina or urethra, vaginal bleeding or discharge, and postcoital bleeding.

Treatment is simple local excision. Recurrence is extremely uncommon.
We presented this case as a rare but important cause of PMB. The lesion is easily treated by local excision. We had expected an endometrial pathology in view of the ultrasound finding of an 11-mm endometrial thickness. It is difficult to explain the ultrasound finding, but the ultrasound findings were on a transabdominal route in a lady with a large BMI.

Conflict of interest There is no actual or potential conflict of interest in relation to this article.

\section{References}

1. Sharma S, Albertazzi P, Richmond I (2006) Vaginal polyps and hormones-is there a link? A case series. Maturitas 53(3):351355

2. Guida M, Di Spiezio Sardo A, Mignogna C, Stefano B, Nappi C (2008) Vaginal fibro-epithelial polyp as cause of postmenopausal bleeding: office hysteroscopic treatment. Gynecol Surg 5:69-70

3. Ramírez Melgar E, Kunhardt Urquiza E, Romero Arauz J, García Barcenas JL (2000) Fibroepithelial polyp of the vagina. Report of a case. Ginecol Obstet Mex 68:368-370 (in Spanish)

4. Nucci MR, Oliva E (2009) Gynecologic pathology. Elsevier Health Sciences, UK, pp 31-33

5. Halvorsen TB, Johannesen E (1992) Fibroepithelial polyps of the vagina: are they old granulation tissue polyps? J Clin Pathol 45:235-240

6. Rollason TP, Byrne P, Williams A (1990) Immunohistochemical and electron microscopic findings in benign fibro epithelial vaginal polyps. J Clin Pathol 43(3):224-229

7. Nucci MR, Young RH, Fletcher CD (2000) Cellular pseudosarcomatous fibroepithelial stromal polyps of the lower female genital tract: an under recognized lesion often misdiagnosed as sarcoma. Am J Surg Pathol 24(2):231-240 